\title{
Tagging to endogenous genes of Plasmodium falciparum using CRISPR/Cas9
}

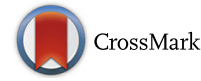

\author{
Dexuan Kuang ${ }^{1 \dagger}$, Jichen Qiao ${ }^{2,3 \dagger}$, Zhou Li ${ }^{4}$, Weiwei Wang ${ }^{4}$, Hui Xia ${ }^{2,3}$, Lubin Jiang ${ }^{4}$, Jiejie Dai ${ }^{{ }^{*}}$, Qiang Fang ${ }^{2,3^{*}}$ \\ and Xueyu Dai ${ }^{4^{*}}$
}

\begin{abstract}
Background: Plasmodium falciparum is the deadliest malaria parasite. Currently, there are seldom commercial antibodies against $P$. falciparum proteins, which greatly limits the study on Plasmodium. CRISPR/Cas9 is an efficient genome editing method, which has been employed in various organisms. However, the use of this technique in $P$. falciparum is still limited to gene knockout, site-specific mutation and generation of green fluorescent protein (GFP) reporter line with disruption of inserted sites.

Results: We have adapted the CRISPR/Cas9 system to add commercial tag sequences to endogenous genes of $P$. falciparum. To add HA or HA-TY1 tags to ck2 $\beta 1$, ck2 $\alpha$ and stk, pL6cs-hDHFR-ck2 $\beta 1 / c k 2 a / s t k$ was constructed, which contained sequences of tags, specific homologous arms, and sgRNA. The $P$. falciparum 3D7 strain was subsequently transfected with pUF1-BSD-Cas9 and pL6cs-hDHFR-ck2 $31 / c k 2 a / s t k$ plasmids via electroporation. After that, BSD and WR99210 drugs were added to the culture to screen parasites containing both plasmids. Twenty days after electroporation, live parasites appeared and were collected to check the tagging by PCR, DNA sequencing, Western blotting and immuno-fluorescence assays. The results showed that the tags were successfully integrated into the Cterminus of these three proteins.
\end{abstract}

Conclusions: We have improved the method to integrate tags to Plasmodium falciparum genes using the CRISPR/ Cas9 method, which lays the foundation for further study of Plasmodium falciparum at the molecular level.

Keywords: Plasmodium falciparum, CRISPR/Cas9, Gene editing, Gene tagging

\section{Background}

Malaria is an infectious disease which presents a serious threat to human life and health. Plasmodium falciparum is the deadliest malaria parasite, which gives rise to many clinical cases around the world each year [1]. Malaria can cause a variety of complications, such as anemia, hepatosplenomegaly, cerebral malaria, miscarriage and death [2-4]. Currently, there is still no effective vaccine to prevent malaria, so the strategy for controlling the disease is dependent on anti-malarial drugs [5-7]. The emergence of artemisinin has

\footnotetext{
* Correspondence: djj@imbcams.com.cn; fq333@sohu.com; xydai@ips.ac.cn †Equal contributors

${ }^{1}$ Institute of Medical Biology, Chinese Academy of Medical Science and Peking Union Medical College, Kunming, Yunnan 650118, China

2Department of Microbiology and Parasitology, Chinese Academy of Medical Science and Peking Union Medical College, Kunming, Yunnan 650118, China ${ }^{4}$ Institut Pasteur of Shanghai, Chinese Academy of Sciences, Shanghai 200031, China

Full list of author information is available at the end of the article
}

effectively controlled the spread of malaria. However, artemisinin-resistant parasites have appeared in Southeast Asia [8-10]. Thus, malaria prevention and therapy remain an important mission.

The study of Plasmodium falciparum at the molecular level can significantly promote the development of malaria vaccines and anti-malarial drugs [11, 12]. Before the emergence of CRISPR/Cas9, single or double-crossover recombination strategies were used to edit genes in $P$. falciparum [13-15]. Briefly, plasmids were used to transfect parasites and kept as episomes [14]. Then several on/off drug selection cycles were carried out to isolate parasites with the desired chromosomal integration event. This method was very inefficient and required months to achieve the desired gene modification [16]. The Streptococcus pyogenes CRISPR/Cas9 system has enhanced gene editing in various organisms [17-19]. Cas9 endonuclease is guided to a specific target DNA site by the single guide RNA (sgRNA) and subsequently induces 
double-strand breaks (DSBs) at this site. The DSBs are then repaired by homologous recombination using donor DNAs since the canonical nonhomologous endjoining (NHEJ) is deficient in Plasmodium [20]. This technique has already been used in P. falciparum for gene knock out, generating single-nucleotide substitutions and a green fluorescent protein (GFP) reporter line with disruption of inserted sites [17, 19, 21], but the adaption of this system for adding tags to $P$. falciparum genes has not been reported yet.

Currently, there are few commercially available antibodies against $P$. falciparum proteins, which greatly limits the study of Plasmodium. Based on CRISPR/Cas9, we adapted this system to add commercial tag sequences (such as HA, TY1) to P. falciparum genes. We show that this tagging strategy is comparatively quick, as it only takes 20 days to achieve transgenic $P$. falciparum, and efficient since we successfully obtained three distinct tagged genes in three trials. This tagging system lays the foundation for molecular studies on Plasmodium falciparum.

\section{Methods}

\section{Plasmid construction and preparation}

Based on pUF1-Cas9 and pL6cs plasmids, kindly provided by Jose-Juan Lopez-Rubio, pUF1-BSD-Cas 9 and pL6cs-hDHFR-ck $2 \beta 1 / \mathrm{ck} 2 \alpha /$ stk plasmids were constructed to create our tagging system.

pUF1-BSD-Cas9 expresses Cas9 endonuclease and blasticidin S deaminase (BSD). The pUF1-Cas9 vector was digested with BamHI and HindIII to remove the yDHODH coding sequences. The BSD coding sequences from pCC4 [22] were amplified by PCR and then inserted in the linearized pUF1-Cas9 using in-fusion cloning kit (Vazyme Biotech, Nanjing, China).

The pL6cs-hDHFR-ck $2 \beta 1 / \mathrm{ck} 2 \alpha /$ stk plasmids, which offer donor DNAs and sgRNAs, were constructed in multiple steps. First, the pL6cs plasmid was digested with $P v u I$ I and $A s c \mathrm{I}$, and the yFCU expression cassette was replaced with the annealed DNA fragment to introduce some new restriction site cassettes (Additional file 1) using in-fusion system to generate pL6cs-hDHFR vector. Next, the left and right homologous arms (i.e. donor DNAs) were amplified separately by PCR from genomic DNA of P. falciparum 3D7 (primers P3/P4 and P5/P6 for ck2 $\beta 1, \mathrm{P} 15 / \mathrm{P} 16$ and $\mathrm{P} 17 / \mathrm{P} 18$ for $\mathrm{ck} 2 \alpha, \mathrm{P} 25 / \mathrm{P} 26$ and P27/P28 for stk).

For pL6cs-hDHFR-ck2 $\beta 1$, the linker-HA motif was amplified from synthesized HA DNA using P7/P8, producing a soft linker, $\mathrm{HA}$ and overlapped sequences to ck2 $\beta 1$ homologous arms. Similarly, for pL6cs-hDHFRck2 $\alpha$ and pL6cs-hDHFR-stk plasmids, the linker-HATY1 motif was amplified from synthesized HA-TY1 DNA using P19/P20 or P29/P30, producing a soft linker,
HA-TY1 and overlapped sequences to $\mathrm{ck} 2 \alpha$ or stk homologous arms.

Then, the 1st bridging PCR was run from linker-HA or linker-HA-TY1 motif mixed with respective left homologous arm (P3/P8 for ck2 $\beta 1$, P15/P20 for ck2 $\alpha$, and P27/ P30 for stk). The 2nd bridging PCR was run using the first bridging PCR product mixed with right homologous arm, respectively (P9/P10 for ck2 $\beta 1, \mathrm{P} 21 / \mathrm{P} 22$ for $\mathrm{ck} 2 \alpha$ and P26/P27 for stk). To prevent the already edited genomic DNA from being recognized and cut again by Cas 9 after successful tagging, DNA sequences which expressed sgRNAs and PAM motif in donor DNAs (i.e. homologous arms) were mismatched to synonymous mutations. For ck $2 \alpha$ and stk, the NGG was close to the stop codon and thus the mismatched mutations were introduced during the PCRs of left homologous arms from genomic DNA. Subsequently, the second bridging PCR products for $\mathrm{ck} 2 \alpha$ and stk were inserted into the pL6cs-hDHFR vector which was linearized with $A s c \mathrm{I}$ and $A f l \mathrm{II}$ using an in-fusion cloning kit to create transitional-pL6cs-hDHFR-ck2 $\beta 1$ and transitional-pL6cs-hDHFR-stk plasmids.

For ck2 $\beta 1$, a mismatched mutation was introduced by extra PCRs using the 2nd bridging PCR product and two pairs of primers separately (P9/P12 and P11/P10). These two PCR products were mixed as template for final PCR using P9/P10 primers. This final DNA fragment was inserted into pL6cs-hDHFR vector linearized with EcoRI and NcoI using the in-fusion system to generate a transitional-pL6cs-hDHFR-ck2 31 plasmid.

The constructs of transitional-pL6cs-hDHFR-ck2 $\beta 1 /$ ck $2 \alpha /$ stk were transformed into competent cells of XL10. The plasmids were then extracted using Plasmid Mini Kit (Qiagen, Dusseldorf, Germany) and checked with restriction enzyme digestion as well as DNA sequencing. After the correct transitional-pL6cs-hDHFRck $2 \beta 1 / \mathrm{ck} 2 \alpha /$ stk plasmids were obtained, these transitional plasmids were linearized with AvrII \& XhoI. The primers $\mathrm{P} 13 / \mathrm{P} 14, \mathrm{P} 23 / \mathrm{P} 24$ and $\mathrm{P} 31 / \mathrm{P} 32$ were used to synthesize the DNA sequences expressing sgRNAs of ck2 $\beta 1$, ck $2 \alpha$ and stk, respectively. After being annealed, DNA fragments of P13/P14, P23/P24, P31/P32 were respectively inserted into linearized transitional-pL6cshDHFR-ck $2 \beta 1 / \mathrm{ck} 2 \alpha /$ stk plasmids using in-fusion kit. The constructs were transformed into competent cells of XL-10 again and then extracted with Plasmid Mini Prep Kit. These final plasmids were confirmed by restriction enzyme digestion and DNA sequencing. The confirmed plasmids were isolated with Plasmid Mega Kit (Qiagen) and further used for electroporation to generate $P$. falciparum transgenic strains.

\section{Plasmodium transfection with plasmids}

Cytomix buffer was made according to the previous report and kept at $-20{ }^{\circ} \mathrm{C}$ [23]. Plasmodium 3D7 was 
cultured in fresh human red blood cells at $37{ }^{\circ} \mathrm{C}, 5 \%$ $\mathrm{CO}_{2}, 5 \% \mathrm{O}_{2}$ in RPMI-1640 medium containing $5 \mathrm{~g} / \mathrm{l}$ Albumax. Parasites were synchronized with Percoll [24]. Fifteen hours after Percoll, the parasites had developed to the ring stage with around 5\% parasitemia. The iRBCs (infected red blood cells) containing parasites were washed twice with $1 \times$ cytomix just before electroporation. The electroporation mixture contained $50 \mu \mathrm{g}$ of pUF1-BSD-Cas9 plasmid $(25 \mu \mathrm{l}), 50 \mu \mathrm{g}$ of pL6cshDHFR-ck $2 \alpha(25 \mu \mathrm{l}), 100 \mu \mathrm{l}$ iRBC, $150 \mu \mathrm{l} 2 \times$ Cytomix, and $100 \mu \mathrm{l} \mathrm{H}_{2} \mathrm{O}$. The same mix formula was used for ck2 $\beta 1$ and stk. Parameters during electroporation performed with GenePulser Xcell (Bio-Rad, Hercules, California, USA) were set as follows: voltage is $310 \mathrm{~V}$, capacity is $950 \mu \mathrm{F}$, electric resistance is infinite; cuvette gap is $2 \mathrm{~mm}$. After electroporation, the parasites were transferred into flasks with medium, and cultured in the incubator with $5 \% \mathrm{O}_{2}, 5 \% \mathrm{CO}_{2}$ at $37{ }^{\circ} \mathrm{C}$. One day after electroporation, the iRBCs were smeared on slides and stained with Giemsa solution; usually, the parasitemia was around $2.5 \%$ at this time. BSD (selection marker encoded by pUF1-BSD-Cas9 plasmid) and WR99210 drugs (selection marker encoded by pL6cs-hDHFRck $2 \beta 1 / \mathrm{ck} 2 \alpha /$ stk plasmid) were freshly added to the culture every two days, to kill those parasites without episomal pUF1-BSD-Cas9 plasmid or pL6cs-hDHFRck $2 \beta 1 / c k 2 \alpha /$ stk. All primer (from P1 to P36) and sgRNA sequences used for constructing plasmids can be seen in Additional file 1.

\section{Confirmation of tagging via PCR and DNA sequencing}

Twenty days after electroporation, live $P$. falciparum appeared and were collected for genomic DNA isolation. A PCR check was carried out with specific primers (P1/P2, as indicated in Fig. 2) and the genomic DNAs as template. After PCR, if live P. falciparum were $100 \%$ tagged, the two drugs were not added into the culturing; if the live $P$. falciparum was NOT $100 \%$ tagged, both drugs were continuously added to the culture until the tagged population reached $100 \%$. The PCR product from wildtype 3D7 genomic DNA was used as negative control of tagging. PCR products were analyzed on agarose gels, and products with the expected size were sent for sequencing to confirm the expected tagging.

\section{Confirmation of tagging via western blotting}

Successfully transgenic parasites were cultured in flasks. When parasitemia passed $5 \%$, iRBCs were collected and incubated with $0.15 \%$ saponin lysis solution on ice for $7 \mathrm{~min}$. After centrifugation, the supernatant was removed. The $P$. falciparum pellet was resuspended in appropriate volume of cell lysate buffer and broken with sonication. After high-speed centrifugation at low temperature, the supernatant was placed in new tubes, mixed with SDS-PAGE loading buffer and denatured at $98{ }^{\circ} \mathrm{C}$ for $5 \mathrm{~min}$. After cooling, the samples were loaded to SDS-PAGE and transferred to PVDF membrane. Integrated Tags were detected by using anti-TY1 antibody (Sigma-Aldrich, Saint Louis, USA) or anti-HA antibody (Abcam, Massachusetts, USA).

\section{Immunofluorescence assay (IFA)}

IFA was performed as previously described [25]. Briefly, $P$. falciparum was synchronized with Percoll and then collected at ring, trophozoite or schizont stage. Infected red blood cells, were fixed with $4 \%$ paraformaldehyde for $20 \mathrm{~min}$, and then washed 3 times with PBS, and permeabilized with $0.15 \%$ Trion X-100. Parasites were incubated overnight at $4{ }^{\circ} \mathrm{C}$ with mouse anti-TY1 antibody (SigmaAldrich, Saint Louis, USA) in PBS buffer containing 5\% BSA (bovine serum albumin). After three washes with PBS, parasites were incubated with goat anti-mouse DyLight 550 antibody (Thermo Fisher Scientific, Waltham, USA) at $37{ }^{\circ} \mathrm{C}$ for $2 \mathrm{~h}$ and washed again 3 times with PBS. Parasites were placed on slides to form a monolayer, air-dried, and then sealed with mounting medium containing DAPI (VECTOR, California, USA). Pictures were taken under Leica S5 confocal microscope.

\section{Results}

\section{Successful construct for three genes}

ck2 $\beta 1$ (PF3D7_1103700), ck2 $\alpha$ (PF3D7_1108400) and stk (PF3D7_0321400) genes, encode casein kinase 2 beta subunit 1 , casein kinase 2 alpha subunit, and a putative protein kinase, respectively. Immunofluorescence assay (IFA), chromatin immunoprecipitation (ChIP) and coimmunoprecipitation (Co-IP) studies were required for these three proteins. However, there are no commercially available antibodies against these proteins. Thus, we decided to add commercial tag sequence to these three genes using CRISPR/Cas9 technology.

The pUF1-BSD-Cas9 plasmid, which offered Cas9 endonuclease and blasticidin $\mathrm{S}$ deaminase (BSD), was constructed from pUF1-Cas9 plasmid by changing drug selection marker from yDHODH into BSD. The plasmids which provide donor DNAs and sgRNA were constructed from the pL6cs plasmid after several modification steps. First, gene-specific DNA cassettes expressing sgRNAs were chosen from sequences around stop codon and inserted to pL6cs vector. Secondly, since tags were fused to the $C$-terminal ends of ck2 $\beta 1, \mathrm{ck} 2 \alpha$ and stk, two $\sim 550$ bp genomic DNA sequences flanking the stop codon were chosen as homologous arms. Then, HA or HA-TY1 tag sequences were fused between the left and the right homologous arm sequences by bridge PCR. Each continuous sequence containing homologous arms and one of the above tags were inserted into pL6cs-hDHFR vector (Fig. 1). To prevent the already 


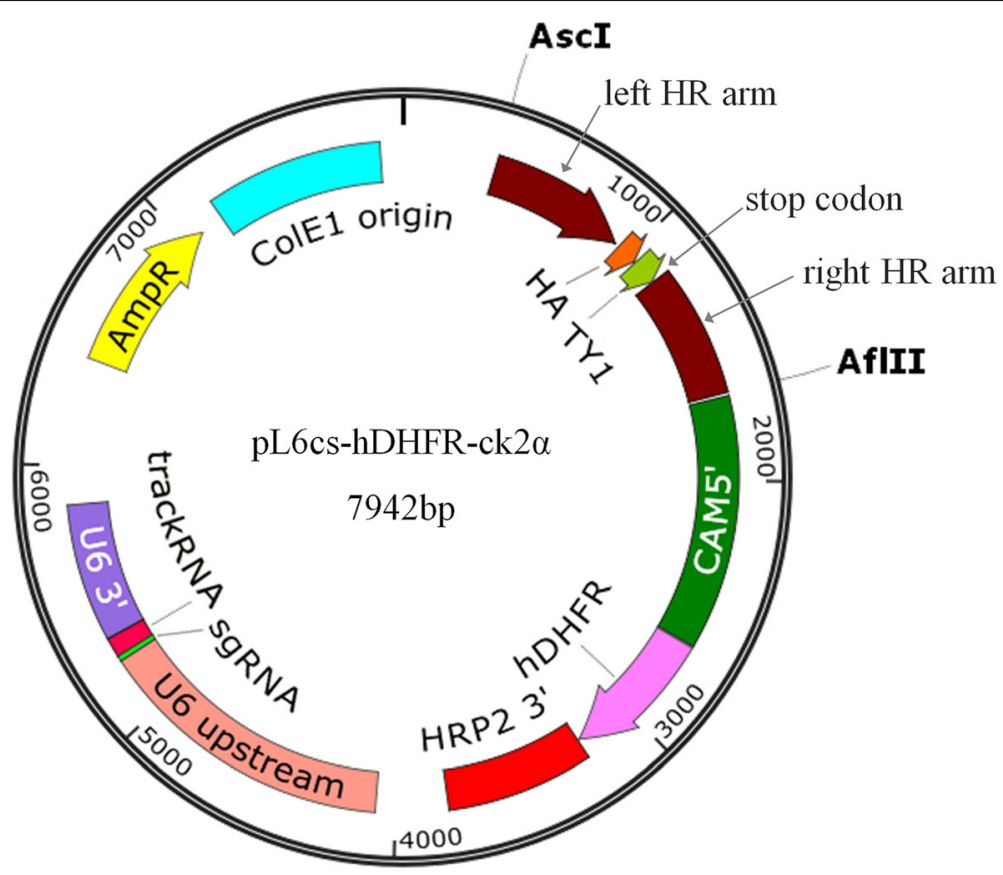

Fig. 1 Plasmid map of pL6cs-hDHFR-ck2a. The HA-TY1 tag coding sequences were placed in the middle of the left and right homologous arms by the bridging PCR method. This continuous DNA fragment was then inserted into the pL6cs-hDHFR vector linearized with Ascl and Aflll by using an in-fusion method. The plasmid map of pL6cs-hDHFR-ck2a was used as a map example. The plasmid maps offering donor DNAs for ck2 $\beta 1$ and stk are similar to this ck2a map, except homologous arms and sgRNA expressing cassettes are gene specific

edited genomic DNA from being recognized and cut again by Cas9 after successful tagging, DNA sequences which expressed sgRNAs and PAM motif in donor DNAs (i.e. homologous arms) was mismatched to synonymous mutation.

All constructs were screened with enzyme digestion and DNA sequencing to ensure that the open reading frame for each gene was correct. After that, P. falciparum 3D7 strain was subsequently transfected with $50 \mu \mathrm{g}$ pL6cs-hDHFR-ck2 $\beta 1 / \mathrm{ck} 2 \alpha /$ stk (donor DNA) and $50 \mu \mathrm{g}$ pUF1-BSD-Cas9 plasmids via electroporation. To select the successful transfection of both plasmids, BSD and WR99210 were added to medium one day after electroporation.

\section{Successful tagging checked by PCR and DNA sequencing}

Around 20 days after electroporation, live parasites could be seen in the culture under selection with both drugs. A portion of the live parasite population was collected for genomic DNA isolation, and a PCR was performed to validate the modification of the gene of interest. In these PCRs, we always used two primers which were designed at the genomic DNA sequences beyond the left and right homologous arms (P1/P2, Fig. 2), to prevent PCR contamination from episomal plasmid template. PCR products were analyzed by agarose electrophoresis. For the three genes tested, the size of the amplification matched the expected DNA length with difference between transgenic strains and wild-type 3D7 (Fig. 3). PCR products were also sequenced to confirm that HA or HA-TY1 tags were successfully integrated into genomic DNA at the carboxy-terminal end of ck2 $\beta 1$, ck $2 \alpha$ and stk. Therefore, CRISPR/Cas9 system was successfully used to add tags into endogenous genes of $P$. falciparum. To prevent the dysfunction of parasite proteins caused by tagging, the amino acid sequence GS-G-S-G-G (G is Glycine, $\mathrm{S}$ is Seronine) was added as a soft linker between the tags and coding sequences of parasite genes.

\section{Successful tagging checked by western blot}

Transgenic parasites that had been checked by PCR and DNA sequencing were further confirmed by Western blot. Theoretical molecular weights of the proteins CK2 $\beta 1-\mathrm{HA}, \mathrm{CK} 2 \alpha-\mathrm{HA}-\mathrm{TY} 1$ and STK-HA-TY1 were $33 \mathrm{KD}, 47.7 \mathrm{KD}$ and $46.8 \mathrm{KD}$, respectively (Fig. 4). Western blot results showed that the molecular weight of STKHA-TY1 protein in SDS-PAGE was the same as its theoretical one. The bands corresponding to CK2 $\beta 1-\mathrm{HA}$ and CK2 $\alpha$-HA-TY1 appeared to be slightly larger than their theoretical molecular weight, which may be caused by post-translational modifications of the two proteins. Therefore, the HA or HA-TY1 tag was successfully added to the $c k 2 \alpha, c k 2 \beta 1$ and stk genes of $P$. falciparum using CRISPR/Cas9 system. The tag was integrated into 


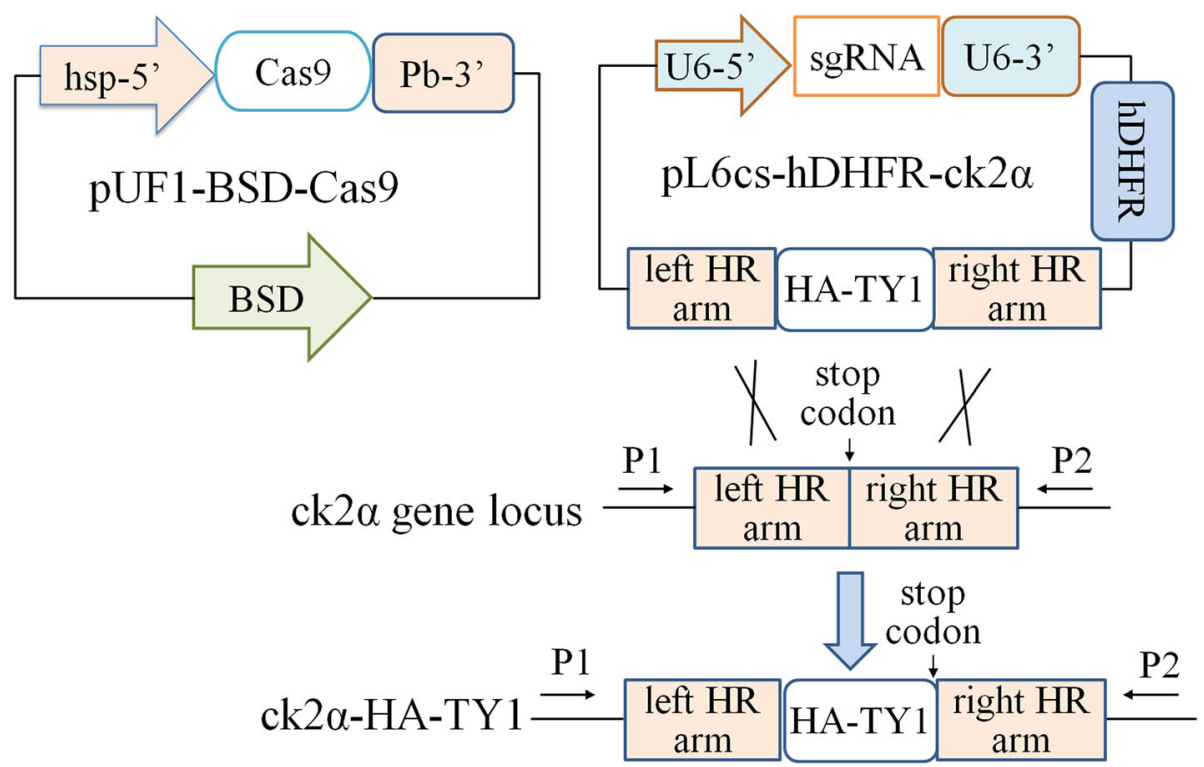

Fig. 2 Schematic illustration of the ck2a tagging principle using CRISPR/Cas9. The HA-TY1 tag sequences are integrated into ck2a gene just before its stop codon through homologous recombination happened at left and right arms. Primers for PCR to check the tagging are labeled as P1 and P2

the proper open reading frames and translated with the endogenous proteins of parasites.

\section{Subcellular localization of STK proteins with immunofluorescence assay}

We used STK-HA-TY1 to analyze the subcellular localization of tagged protein. Transgenic parasites containing STK-HA-TY1 were synchronized and collected at ring, trophozoite and schizont stage. Parasites were fixed and then incubated with anti-TY1 antibody. The immunofluorescence assay (IFA) showed that STK proteins localized in the nucleus as well as at the nuclear periphery during ring stage. The localization of STK protein during trophozoite and schizont stages is similar to the distribution pattern of ring stage but with weaker signal at the nucleus (Fig. 5). This result indicated that HA-TY1 tag was successfully integrated to endogenous STK protein, and subcellular localization of STK protein could be vividly viewed using IFA. HA tag to C-terminus of Pfck $2 \alpha$ or Pfck $2 \beta 1$ has been reported using episomes or old recombination method; subsequent IFAs show that these two tagged proteins localize to both cytoplasmic and nuclear compartments of parasite [26, 27].

\section{Discussion}

The molecular study of $P$. falciparum can promote the development of malaria vaccines and anti-malarial drugs. However, only a few specific antibodies against $P$.

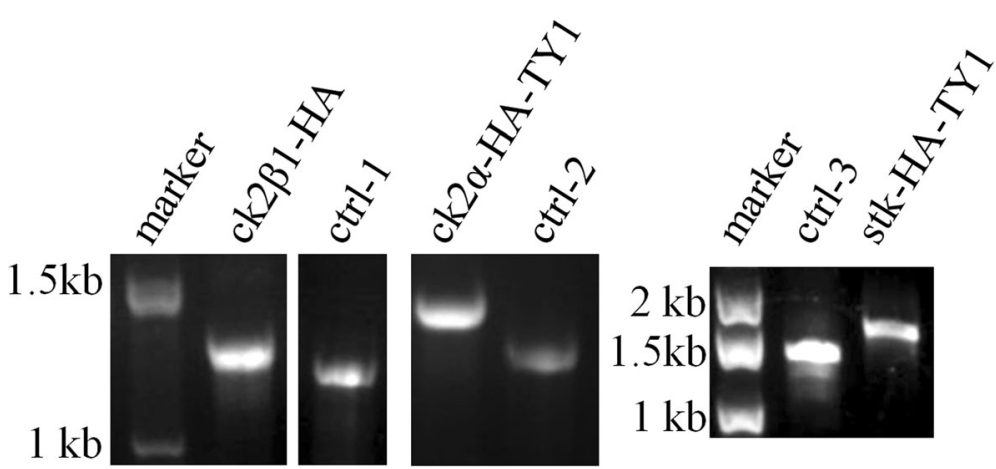

Fig. 3 Genomic DNA PCR to confirm the tagging. Using genomic DNA as the template and specific primers of each gene (P33/P34 for ck2 31 , P1/P2 for ck2a, P35/P36 for stk), the products obtained by PCR were separated on an agarose gel. The negative control was the PCR product obtained from 3D7 genomic DNA. The PCR product sizes of ctrl-1, ctrl-2 and ctrl-3, were 1175 bp, 1437 bp, and 1390 bp, respectively. After tagging, the PCR product sizes of transgenic ck2ß1-HA, ck2a-HA-TY1 and stk-HA-TY1 were 1280 bp, 1650 bp, and 1603 bp, respectively 

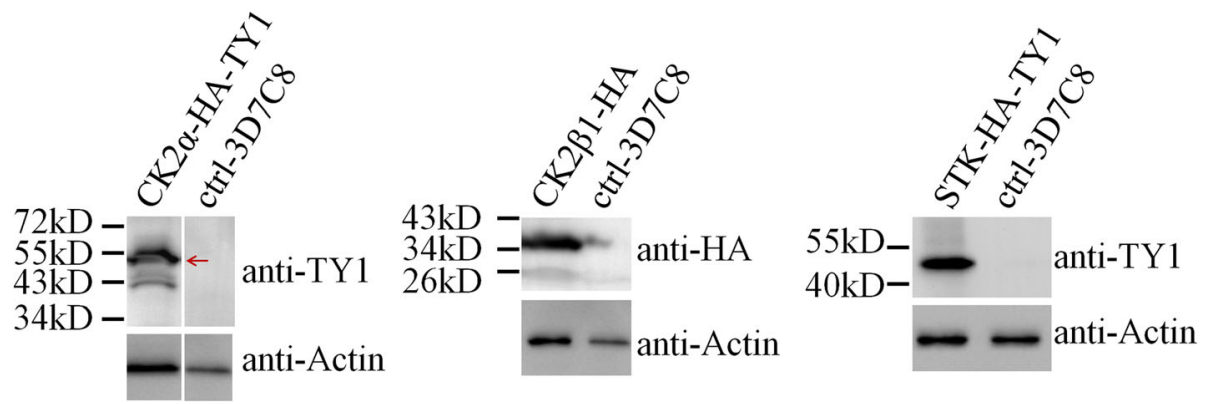

Fig. 4 Western blot to confirm the tagging. The tags added to CK2a, CK2 31 and STK were HA-TY1, HA, HA-TY1, respectively. Therefore, mouse anti-TY1, rabbit anti-HA and mouse anti-TY1 were 1st antibodies used for the Western Blot of CK2a-HA-TY1, CK2 $\beta 1-H A$, STK-HA-TY1, to confirm the successful tagging. The same lysate samples were also separated on SDS-PAGE and blotted with mouse anti-Actin as the loading control. HRP-goat anti-mouse and HRP-goat anti-rabbit were the second antibodies

falciparum proteins are currently available, which greatly limits molecular studies. Before the emergence of CRISPR/Cas9, the old method to edit P. falciaprum genes involved single or double-crossover recombination which was very inefficient and time-consuming (required several months). Recently, CRISPR/Cas9 has been used for gene editing in various organisms including Plasmodium [17-19]. The Cas9 endonuclease is guided to target DNA site by a sgRNA and induces DSBs at this site. Then the induced DSBs is repaired by homologous recombination using donor DNAs. In Plasmodium, the double-strand break repair by homologous recombination is largely favored since canonical NHEJ is deficient in this organism [20]. Up to now, the application of CRISPR/Cas9 system in P. falciparum was limited to gene knock out, generating genome mutation or GFP reporter parasite line with disruption of inserted sites $[17,19,21]$. A recently improved CRISPR/Cas9 system contains Cas9 nuclease, sgRNA, and a selectable marker in one plasmid while homologous arms (donor DNA fragments) without selectable marker in another plasmid. This modified system is used for DNA disruption, where promoter, 3'-UTR and GFP or other tag coding sequences are inserted to disrupt the loci instead of drug selection marker. Thus, this new system is saving one drug selection marker for consecutive gene manipulations in P. falciparum [28]. Efficient tagging to Plasmodium yoelii genes using CRISPR/Cas9 has been reported [29]. Here, we adapt the CRISPR/Cas9 system to add tags to P. falciparum

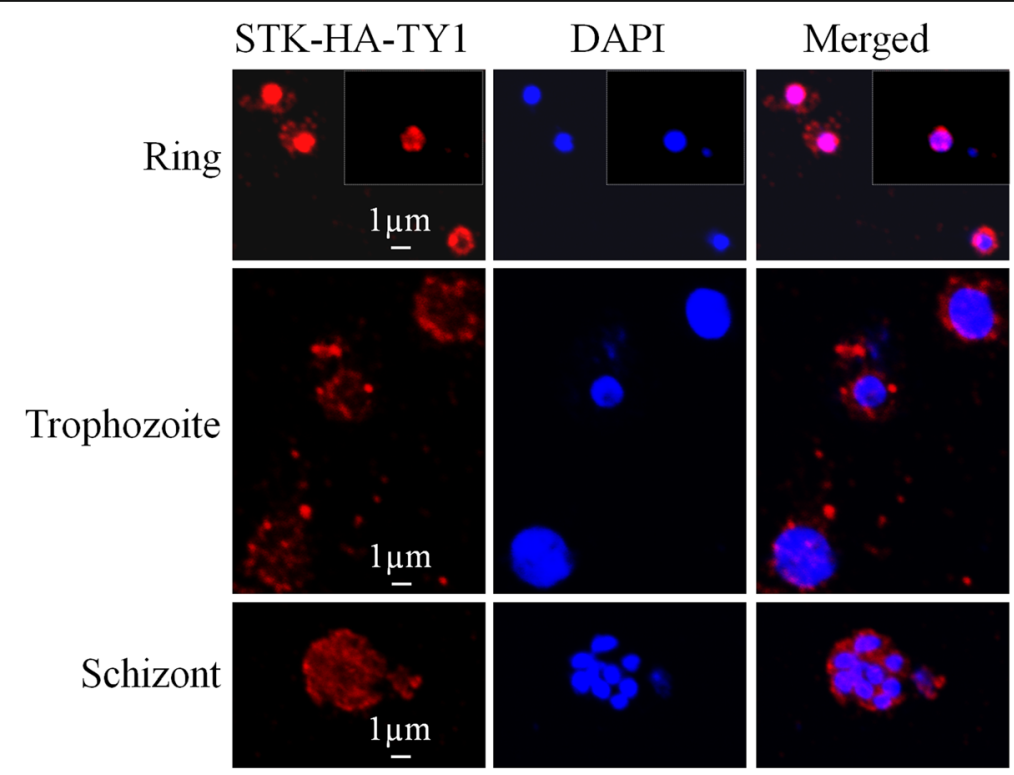

Fig. 5 Subcellular localization of STK protein by immunofluorescence assay. Parasites at ring, trophozoite and schizont stages were collected and incubated with mouse anti-TY1 antibody and then 2nd antibody. The red fluorescence represents STK-HA-TY1; the blue fluorescence is DAPI, representing the parasite nucleus. In the images at top line, the picture highlighted by square with dashed line is IFA of a nucleus taken under other view 
endogenous genes. By using our adapted system, transgenic parasites could be achieved in $\sim 20$ days, significantly shorting the required time for gene tagging. Additionally, we were able to obtain three distinct tagged genes in three trials. With these tagged proteins, many molecular assays such as ChIP, IP and IFA become easy to perform.

\section{Conclusions}

In summary, an efficient method using CRISPR/Cas9 was adapted to add tag sequences to three endogenous genes of $P$. falciparum, which provided a good example for editing parasite genes as well as laid a foundation for the molecular studies of CK2 $\alpha$, CK2 $2 \beta 1$ and STK proteins. This will be beneficial for our understanding of Plasmodium biology and consequently could improve the development of anti-malaria vaccines and drugs.

\section{Additional file}

Additional file 1: $P C R$ primers and sgRNAs used for plasmid construction. (PDF $78 \mathrm{~kb}$ )

\section{Abbreviations}

BSD: blasticidin S deaminase; ChIP: chromatin immunoprecipitation; CK2a: casein kinase 2 alpha subunit; CK2 $\beta 1$ : casein kinase 2 beta subunit 1; Co-IP: co-immunoprecipitation; DSB: double-strand break; HA: human influenza hemagglutinin tag; hDHFR: human dihydrofolate reducatase; IFA: immunofluorescence assay; IP: immunoprecipitation; iRBCs: infected red blood cells; sgRNA: single guide RNA; STK: a putative protein kinase which recognizes Ser/Thr motif; TY1: TY1 tag

\section{Acknowledgements}

We thank Jose-Juan Lopez-Rubio for kindly providing the pUF1-Cas9 and pL6cs plasmids. We thank Dr Nicolas Gilbert and Daphne Ko for their critical comments on this manuscript.

\section{Funding}

This study was supported in part by the National Natural Science Foundation of China (No. 31571345), the Scientific Research Innovation Team Project of Anhui Colleges and Universities (2016-40), the Anhui Provincial Natural Science Foundation (1308085MH160), the Training Project for Provincial Academic Technology Leaders in Anhui Colleges and Universities (2014053), the Yunnan science and technology plan project (2016HB006).

\section{Availability of data and materials}

All data generated or analysed during this study are included in this published article and its supplementary information files.

\section{Authors' contributions}

$X D, L J, J D$ and QF designed research. DK, JQ, ZL and WW performed research. XD, LJ, JD, HX and QF analyzed data; XD wrote the paper. All authors read and approved the final manuscript.

\section{Ethics approval and consent to participate}

Not applicable.

\section{Consent for publication}

Not applicable.

\section{Competing interests}

The authors declare that they have no competing interests.

\section{Publisher's Note}

Springer Nature remains neutral with regard to jurisdictional claims in published maps and institutional affiliations.

\section{Author details}

${ }^{1}$ Institute of Medical Biology, Chinese Academy of Medical Science and Peking Union Medical College, Kunming, Yunnan 650118, China.

${ }^{2}$ Department of Microbiology and Parasitology, Chinese Academy of Medical Science and Peking Union Medical College, Kunming, Yunnan 650118, China

${ }^{3}$ Anhui Key Laboratory of Infection and Immunity at Bengbu Medical College, Bengbu 233030, China. ${ }^{4}$ Institut Pasteur of Shanghai, Chinese Academy of Sciences, Shanghai 200031, China.

Received: 14 June 2017 Accepted: 14 November 2017

Published online: 02 December 2017

\section{References}

1. Murray CJ, Ortblad KF, Guinovart C, Lim SS, Wolock TM, Roberts DA, et al. Global, regional, and national incidence and mortality for HIV, tuberculosis, and malaria during 1990-2013: a systematic analysis for the global burden of disease study 2013. Lancet. 2014;384(9947):1005-70.

2. Bhaumik S. Malaria funds drying up: world malaria report 2012. Natl Med J India. 2013;26(1):62.

3. WHO, World Malaria Report 2015. Geneva: World Health Organization; 2015.

4. White NJ. Malaria: a molecular marker of artemisinin resistance. Lancet. 2014;383(9927):1439-40.

5. Cowman AF, Healer J, Marapana D, Marsh K. Malaria: biology and disease. Cell. 2016;167(3):610-24

6. Kassegne K, Abe EM, Chen JH, Zhou XN. Immunomic approaches for antigen discovery of human parasites. Expert Rev Proteomics. 2016;13(12): 1091-101.

7. Norahmad NA, Mohd Abd Razak MR, Abdullah NR, Sastu UR, Imwong M, Muniandy PK, et al. Prevalence of Plasmodium falciparum molecular markers of antimalarial drug resistance in a residual malaria focus area in Sabah, Malaysia. PLoS One. 2016:11(10):e0165515.

8. Grist EP, Flegg JA, Humphreys G, Mas IS, Anderson TJ, Ashley EA, et al. Optimal health and disease management using spatial uncertainty: a geographic characterization of emergent artemisinin-resistant Plasmodium falciparum distributions in Southeast Asia. Int J Health Geogr. 2016:15(1):37.

9. Lin JT, Juliano JJ, Wongsrichanalai C. Drug-resistant malaria: the era of ACT. Curr Infect Dis Rep. 2010;12(3):165-73.

10. Phyo AP, Nkhoma S, Stepniewska K, Ashley EA, Nair S, McGready R, et al. Emergence of artemisinin-resistant malaria on the western border of Thailand: a longitudinal study. Lancet. 2012;379(9830):1960-6.

11. Dorado EJ, Okoth SA, Montenegro LM, Diaz G, Barnwell JW, Udhayakumar V et al. Genetic characterisation of Plasmodium falciparum isolates with deletion of the pfhrp2 and/or pfhrp3 genes in Colombia: the Amazon region, a challenge for malaria diagnosis and control. PLoS One. 2016:11(9): e0163137.

12. Jiang L, Gaur D, Mu J, Zhou H, Long CA, Miller LH. Evidence for erythrocytebinding antigen 175 as a component of a ligand-blocking blood-stage malaria vaccine. Proc Natl Acad Sci USA. 2011;108(18):7553-8.

13. Crabb BS, Cooke BM, Reeder JC, Waller RF, Caruana SR, Davern KM, et al. Targeted gene disruption shows that knobs enable malaria-infected red cells to cytoadhere under physiological shear stress. Cell. 1997;89(2):287-96.

14. Crabb BS, Cowman AF. Characterization of promoters and stable transfection by homologous and nonhomologous recombination in Plasmodium falciparum. Proc Natl Acad Sci USA. 1996:93(14):7289-94.

15. Wu Y, Kirkman LA, Wellems TE. Transformation of Plasmodium falciparum malaria parasites by homologous integration of plasmids that confer resistance to pyrimethamine. Proc Natl Acad Sci USA. 1996;93(3):1130-4

16. Crabb BS, Rug M, Gilberger TW, Thompson JK, Triglia T, Maier AG, et al. Transfection of the human malaria parasite Plasmodium falciparum. Methods Mol Biol. 2004;270:263-76.

17. Ghorbal M, Gorman M, Macpherson CR, Martins RM, Scherf A, Lopez-Rubio JJ. Genome editing in the human malaria parasite Plasmodium falciparum using the CRISPR-Cas9 system. Nat Biotechnol. 2014:32(8):819-21.

18. Jinek M, Chylinski K, Fonfara I, Hauer M, Doudna JA, Charpentier EA. Programmable dual-RNA-guided DNA endonuclease in adaptive bacterial immunity. Science. 2012;337(6096):816-21. 
19. Wagner JC, Platt RJ, Goldfless SJ, Zhang F, Niles JC. Efficient CRISPR-Cas9mediated genome editing in Plasmodium falciparum. Nat Methods. 2014; 11(9):915-8

20. Kirkman LA, Lawrence EA, Deitsch KW. Malaria parasites utilize both homologous recombination and alternative end joining pathways to maintain genome integrity. Nucleic Acids Res. 2014;42(1):370-9.

21. Mogollon CM, van Pul FJ, Imai T, Ramesar J, Chevalley-Maurel S, de Roo GM, et al. Rapid generation of marker-free $P$. falciparum fluorescent reporter lines using modified CRISPR/Cas9 constructs and selection protocol. PLoS One. 2016;11(12):e0168362.

22. Maier AG, Braks JA, Waters AP, Cowman AF. Negative selection using yeast cytosine deaminase/uracil phosphoribosyl transferase in Plasmodium falciparum for targeted gene deletion by double crossover recombination. Mol Biochem Parasitol. 2006;150(1):118-21.

23. Jiang L, Mu J, Zhang $\mathrm{Q}, \mathrm{Ni} T$, Srinivasan $\mathrm{P}$, Rayavara $\mathrm{K}$, et al. PfSETvs methylation of histone H3K36 represses virulence genes in Plasmodium falciparum. Nature. 2013;499(7457):223-7.

24. Rivadeneira EM, Wasserman M, Espinal CT. Separation and concentration of schizonts of Plasmodium falciparum by Percoll gradients. J Protozool. 1983; 30(2):367-70.

25. Tonkin CJ, van Dooren GG, Spurck TP, Struck NS, Good RT, Handman E, et al. Localization of organellar proteins in Plasmodium falciparum using a novel set of transfection vectors and a new immunofluorescence fixation method. Mol Biochem Parasitol. 2004;137(1):13-21.

26. Dastidar EG, Dayer G, Holland ZM, Dorin-Semblat D, Claes A, Chene A, et al. Involvement of Plasmodium falciparum protein kinase CK2 in the chromatin assembly pathway. BMC Biol. 2012;10:5.

27. Holland Z, Prudent R, Reiser JB, Cochet C, Doerig C. Functional analysis of protein kinase CK2 of the human malaria parasite Plasmodium falciparum. Eukaryot Cell. 2009;8(3):388-97.

28. Lu J, Tong Y, Pan J, Yang Y, Liu Q, Tan X, et al. A redesigned CRISPR/Cas9 system for marker-free genome editing in Plasmodium falciparum. Parasit Vectors. 2016:9:198.

29. Zhang C, Gao H, Yang Z, Jiang Y, Li Z, Wang X, et al. CRISPR/Cas9 mediated sequential editing of genes critical for ookinete motility in Plasmodium yoelii. Mol Biochem Parasitol. 2017;212:1-8.

\section{Submit your next manuscript to BioMed Central and we will help you at every step:}

- We accept pre-submission inquiries

- Our selector tool helps you to find the most relevant journal

- We provide round the clock customer support

- Convenient online submission

- Thorough peer review

- Inclusion in PubMed and all major indexing services

- Maximum visibility for your research

Submit your manuscript at www.biomedcentral.com/submit 